\title{
Research Paper \\ Does Population Aging Affect Income Inequality in Iran?
}

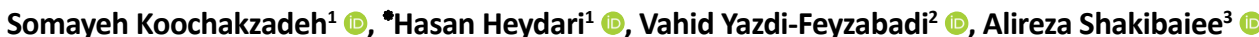

1. Department of Economics, Faculty of Management and Economics, Tarbiat Modares University, Tehran, Iran.

2. Health Services Management Research Center, Institute for Futures Studies in Health, Kerman University of Medical Sciences, Kerman, Iran.

3. Department of Economics, Faculty of Management and Economics, Shahid Bahonar University, Kerman, Iran.

\begin{tabular}{c|c|c|}
$\begin{array}{c}\text { Use your device to scan } \\
\text { and read the article online }\end{array}$ \\
Iran? (Persian)]. Iranian Journal of Ageing. 2021; 16(3):396-411. http://dx.doi.org/10.32598/sija.2021.16.3.3113.1 \\
doisttp://dx.doi.org/10.32598/sija.2021.16.3.3113.1
\end{tabular}

\section{(c) (1) (3)}

Received: 22 Nov 2020 Accepted: 17 Feb 202 Available Online: 01 Oct 2021

Keywords: Aging, Income inequality, IRAN

\section{ABSTRACT}

Objectives The aging of the population in recent years in Iran has raised concerns among policymakers in various fields. Some economic theories consider the changes in the age structure and population aging as one of the important issues affecting the income inequality of societies. This study aims to assess the effect of population aging on the income inequality in Iran.

Methods \& Materials This study was conducted in 2020 using economic data from 1970 to 2018 extracted from the websites of the Statistical Center of Iran and the World Development Indicators. First, a mode for income inequality was presented and demographic variables were considered in the model. Then, its coefficients were estimated using the AutoRegressive Distributed Lag method in Eviews 10 software. To evaluate the speed of adjustment from the short-run to the long-run equilibrium, the Error Correction Model (ECM) was estimated.

Results The increase in aging population led to increased income inequality in the long run such that $1 \%$ increase in aging index caused a $0.14 \%$ increase in Gini index $(P<0.05)$. The literacy rate, trade openness, and the square of Gross Domestic Product (GDP) per capita had a negative significant effect, and GDP per capita and the Consumer Price Index had a positive significant effect on income inequality, both in the short and long term. The ECM value was reported $44.8 \%$.

Conclusion The aging of the population increases income inequality in Iran. Policymakers should reduce income inequality by investing in education and expansion of trading with other countries as well as stabilizing economic conditions, controlling inflation, and planning to reduce the effects of aging.

$\begin{array}{ll}\text { Extended Abstract } & \begin{array}{l}\text { aging of the population [1]. According to statistics published } \\ \text { by international organizations and the censuses conducted by } \\ \text { the Statistics Center of Iran, the Iranian population is aging. } \\ \text { One of the sectors that is most affected by the demographic } \\ \text { 1. Introduction }\end{array} \\ \begin{array}{l}\text { he process of economic development, along } \\ \text { with the advances in the field of health, has } \\ \text { aging can reduce the active population and, consequently, re- } \\ \text { led to an increase in life expectancy in dif- } \\ \text { duce labor supply. Reduction in labor supply affects produc- } \\ \text { terent parts of the world. On the other }\end{array} & \begin{array}{l}\text { tion costs, economic growth and income inequality [4]. This } \\ \text { hand, the fertility rate has decreased in dif- }\end{array}\end{array}$

* Corresponding Author:

Hasan Heydari, PhD.

Address: Department of Economics, Faculty of Management and Economics, Tarbiat Modares University, Tehran, Iran.

Tel: +98 (912) 1546150

E-mail: hassan.heydari@modares.ac.ir 


\section{Methods}

In this study, data were collected using the library method and those related to Iran's economy. Based on the studies on modeling the effect of aging on income inequality, important demographic and economic variables affecting income inequality are: Aging, Literacy, Gross Domestic Product (GDP), Consumer Price Index (CPI), and trade openness [8, $9,11,33,41]$. Gini index was used as a variable indicating the status of income inequality in Iran. The income inequality Equation 1 is defined as follows:

1) LGINI $_{t}=\alpha+\beta_{1} \quad$ LAgeing $_{\mathrm{t}}+\beta_{2} \quad$ LLiteracy $_{\mathrm{t}}+\beta_{3} \quad \mathrm{LGDP}_{\mathrm{t}}+\beta_{4}$ $\left(\text { LGDP }_{t}\right)^{2}+\beta_{5}$ LCPI $_{t}+\beta_{6}$ LOpenness $_{t}+\beta_{7}$ Du $+\varepsilon_{t}$

Where, LGINI= logarithm of income inequality, LAgeing= Logarithm of aging index (population aged $>65$ / population aged $<15$ ); LLiteracy $=$ Logarithm of literacy rate (enrollment rate in the second academic year), LCPI= Logarithm of CPI, LOpenness $=$ Logarithm of trade openness index (sum of exports and imports / total GDP), LGDP= Logarithm of GDP, and=square of logarithm of GDP. The last two variables were used to test the Environmental Kuznets Curve (EKC) hypothesis. In the Equation 1, Du represents the years of Islamic Revolution and Iran-Iraq war. Data and information related to all variables were extracted from the websites of the Statistics Center of Iran and the World Development Indicators (WDI). All data were reported annually. After determining the income inequality pattern, its coefficients were estimated using AutoRegressive Distributed Lag (ARDL) model in Eviews 10 software. Moreover, in order to evaluate the speed of adjustment from the short-term equilibrium to the long-run equilibrium, the Error Correction Model (ECM) was estimated.

\section{Results}

First, in order to prevent false regression, the stationary of the data was tested. Augmented Dickey-Fuller (ADF) test was used for this purpose. The results showed that except for LLiteracy and LOpenness, which were stationary, all other variables became stationary by differencing once. Given that the variables had values as 0 or 1 , the ARDL method can be used to estimate the model. The results of the showed that the increase in the aging population, both in short and long periods could significantly increase income inequality such that, in the long run, a $1 \%$ increase in the aging index led to a $0.14 \%$ increase in Gini coefficient $(\mathrm{P}<0.05)$. Other variables in the model also had significant changes. The increase in CPI also increases income inequality, while the increase in literacy improves income equality. GDP per capita in the long run had a negative impact on income distribution and exacerbated income inequality. The square of GDP per capita had also a negative value. Thus, in the long run, the EKC hypothesis is confirmed. This is acceptable given that Iran is a developing country; according to EKC hypothesis, the relationship between income inequality and income per capita is positive at the initial stages of economic development, and becomes negative at the final stages. The Du had a positive significant impact on income inequality. Coefficient of the ECM also had a significance value. The ECM coefficient indicated that $44.8 \%$ of the income disequilibrium was corrected from the short run to the long run; almost in two periods, a large part of the short-run error was compensated and the model moved towards a long-run equilibrium. Negative value of the ECM coefficient indicated that any long-run disequilibrium moves towards equilibrium. The values $<1$ indicated the convergence of the model in the short run.

\section{Discussion and Conclusion}

The increase in the aging population, which is the result of a decrease in the birth rate and an increase in survival probability, has intensified income inequality in Iran during 1970-2018. Other effective factors are literacy rate, GDP per capita, CPI, and trade openness. As the country's aging population grows in the coming years, population aging is a potential factor for increased income inequality. Therefore, policymakers should plan to reduce the adverse effects of aging on income inequality, such as increased retirement age and increased participation rate of the elderly in the labor market, by investing in workers' education (education based on current and future needs of the labor market), expansion of trading with other countries, stabilizing economic conditions, and controlling inflation.

\section{Ethical Considerations}

\section{Compliance with ethical guidelines}

This study was approved by the Ethics Committee of Tarbiat Modares University (Code: IR.MODARES. REC.1399.127).

\section{Funding}

This study was extracted from the $\mathrm{PhD}$. dissertation of first author at the Department of Economics, Faculty of Management and Economics, Tarbiat Modares University.

\section{Authors' contributions}

All authors equally contributed to preparing this article.

\section{Conflicts of interest}

The authors declared no conflict of interest. 
This Page Intentionally Left Blank 


\section{آيا سالمندى جمعيث بر ثابر ابرى درآمدى در ايران ثأثير تخذار است؟}

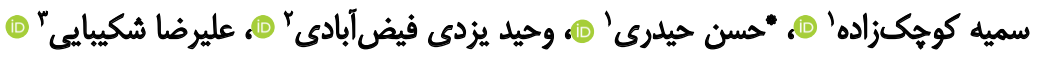

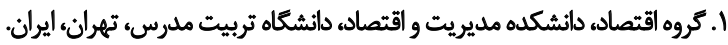

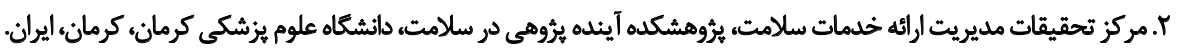

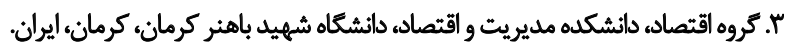

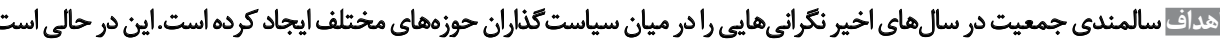

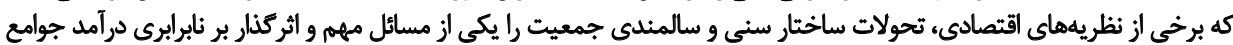

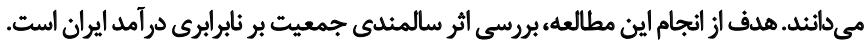

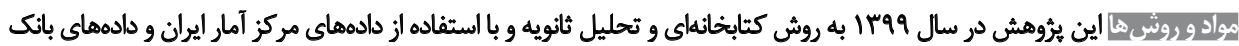

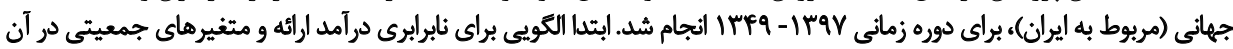

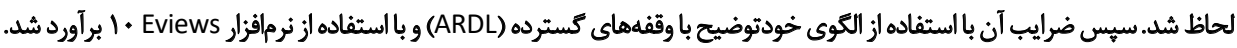

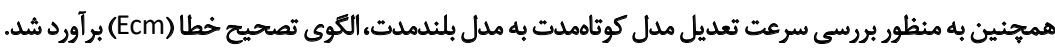

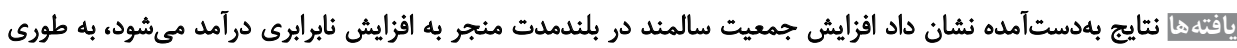

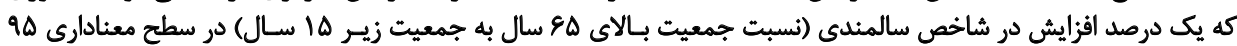

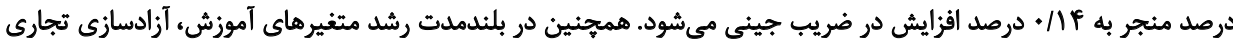

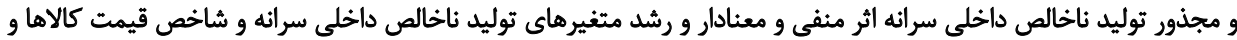

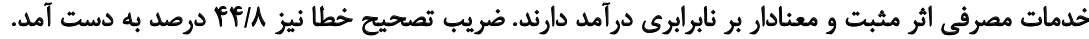

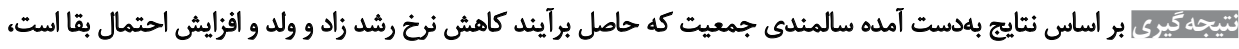

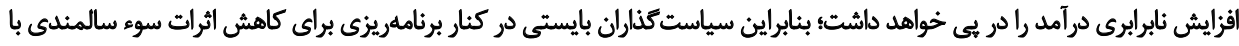

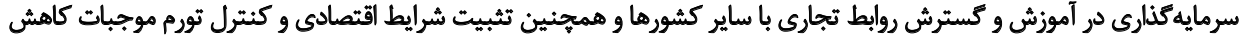

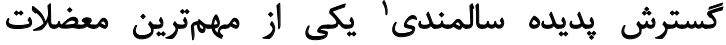

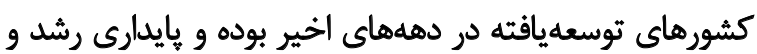

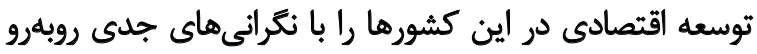

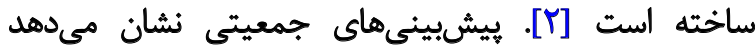

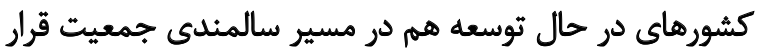

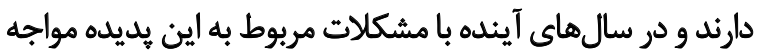

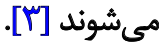
بر اساس آمارهاى منتشرشده توسط سازمانهاى بينالمللى و

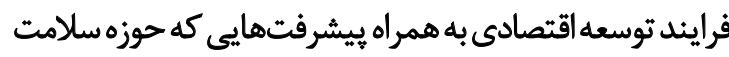

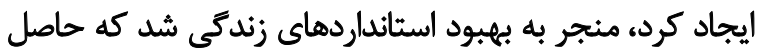

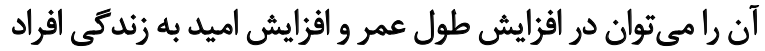

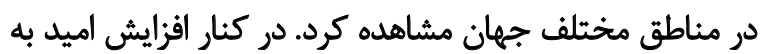

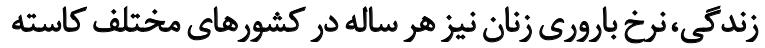

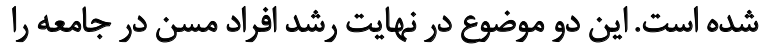

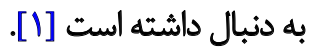

1. Ageing

$$
\text { … }
$$

* نويسئده مسئول:

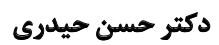

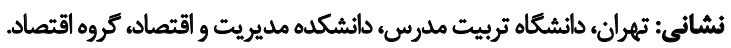

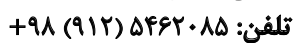

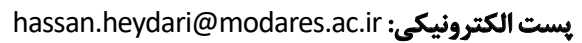


توامل تأثير كذار بر توزيع درآمد در مورد تأثير رشد اقتصادى بر

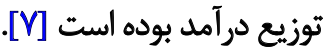

بحث در اين زمينه با فرضيه (u) معكوس) كوزنتس آناز شد.

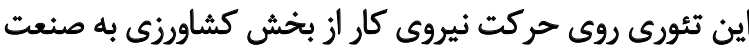

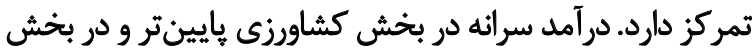

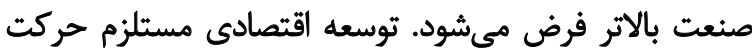
نيروى كار و سرمايه از بخش كشاورزى به بخش صنش صنعت بود. طبق نظريه كوزنتس، در مراحل اوليه توسعه، رابطه نابرابرى

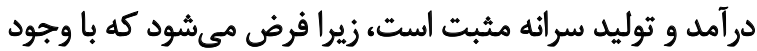

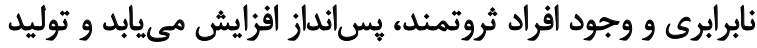

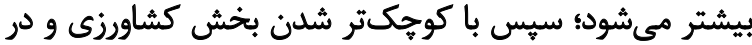

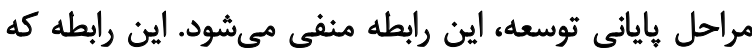

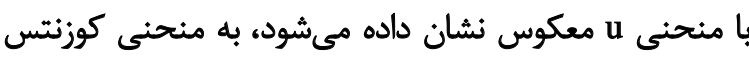

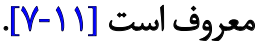

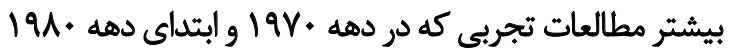

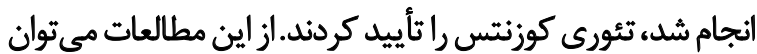

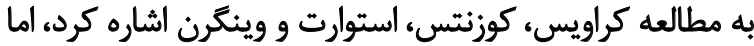

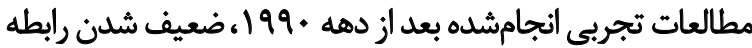

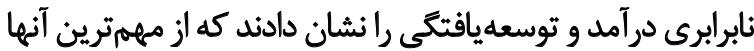

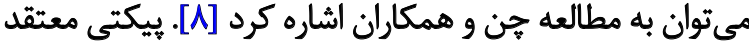

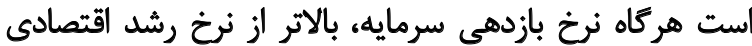

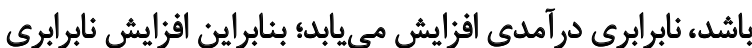

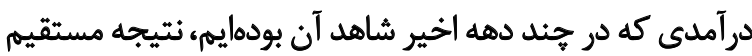

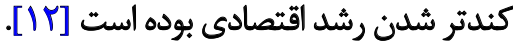

بدبينى مالتوس،بنيان حضور مثغيرهاى جمعيتى در اقتصاد

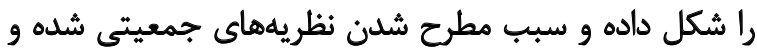

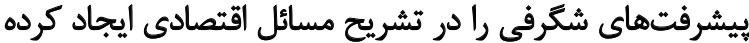

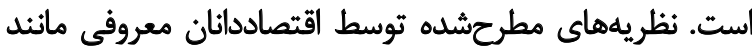

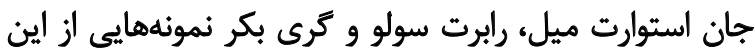

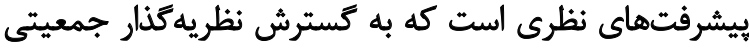

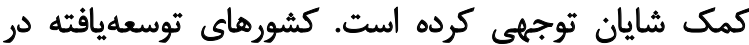

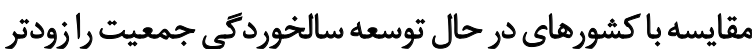

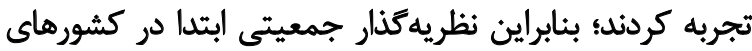

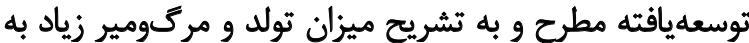

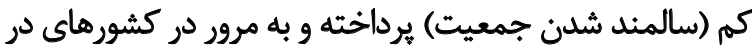

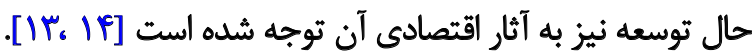

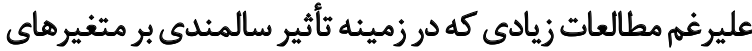

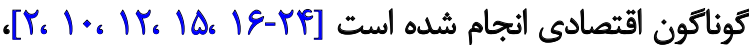

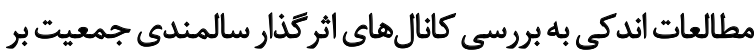
نابرابرى در آمد يرداختهانداندي

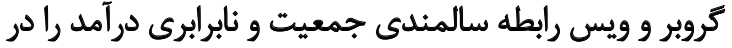

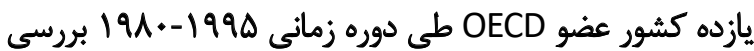

همجنين سرشمارىهاى انجامشده توسط مركز آمار ايران، ساختار

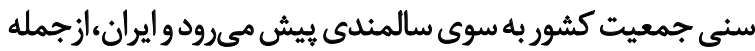

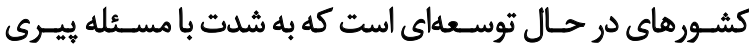

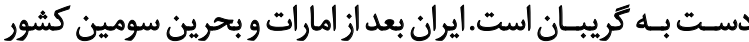

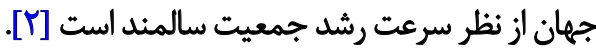
كشور ايران در سه دهه اخير تحولات جمعيتى جشمعيرى

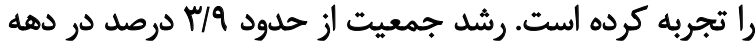

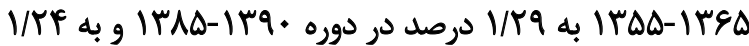

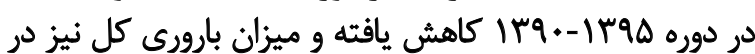

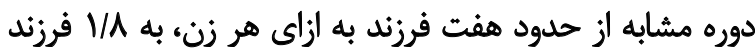

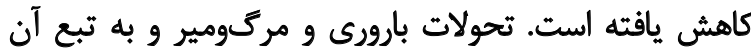

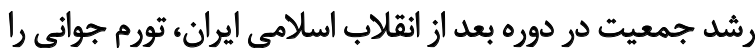

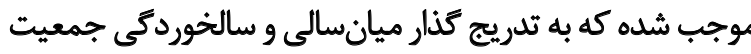

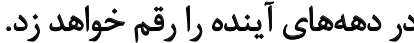

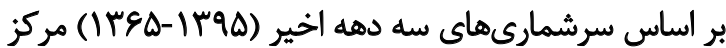

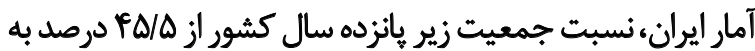

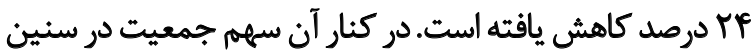

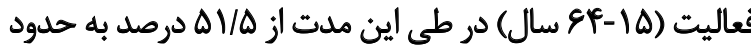

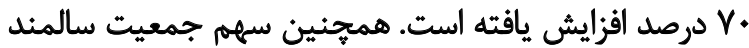

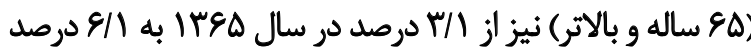

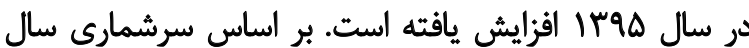

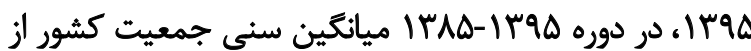

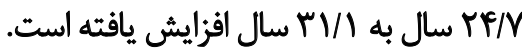

يكى از بخشهايى كه بيشترين اثريذيرى از ساختار جمعيتى

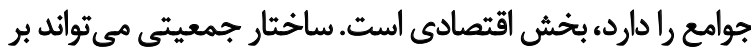

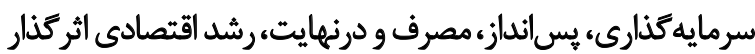

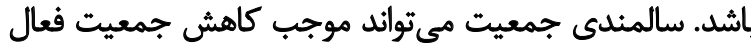

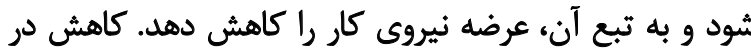

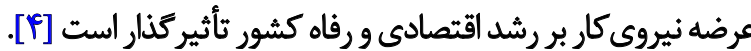

يكى ديكر از يايامدهاى مهم سالمندى جمعيت ايجاد تغييرات

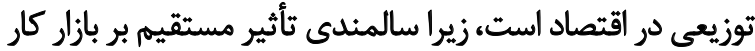

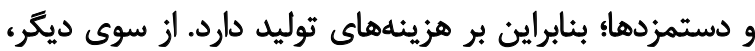

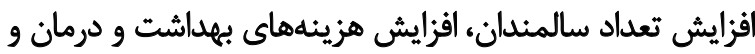

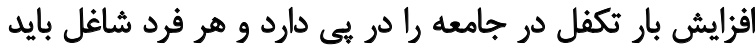

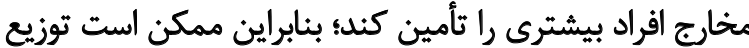

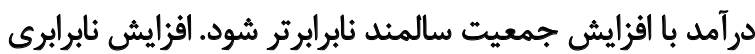

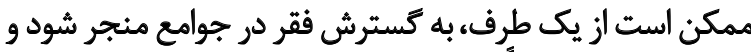

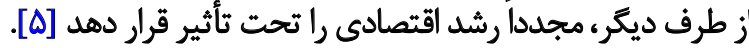
در جيند دهه كُدُشته نابرابرى درآمد در جهان افزايش يافته است.

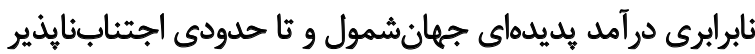

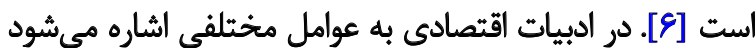
كه بر نابرابرى مؤثر هستند. اولين مطالعات انجامشده در زمينه 


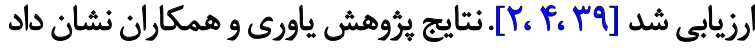

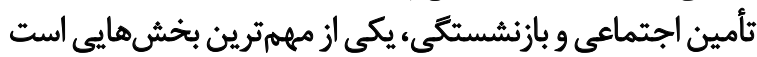

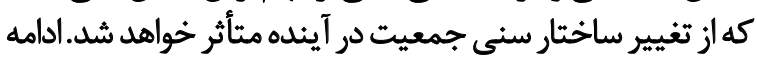

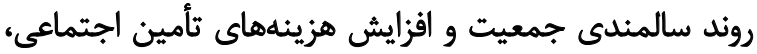

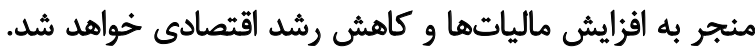

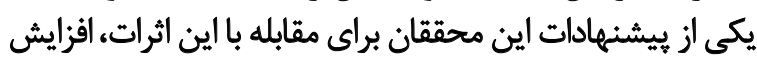

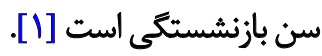

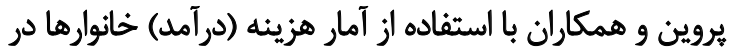

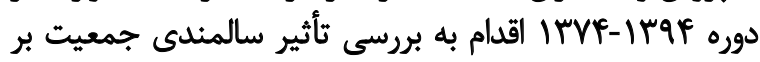

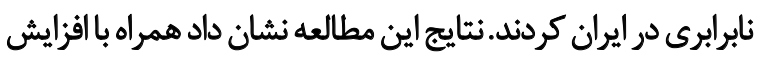

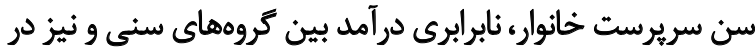

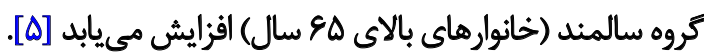

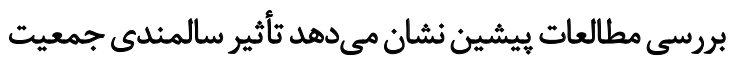

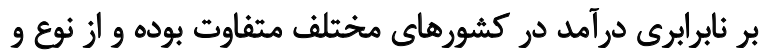

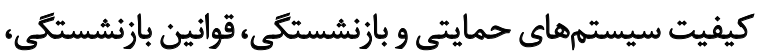

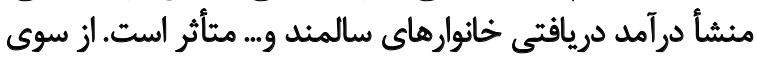

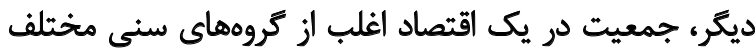

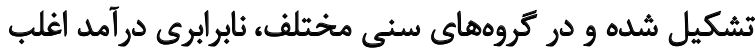

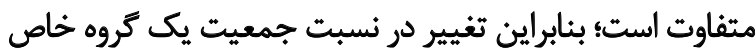

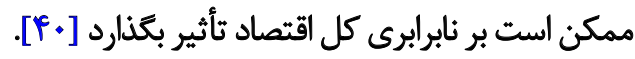
زمانى كه تنها منبع درآمد خانوار حقوق و دستمزد باشد، در

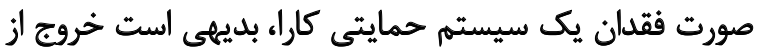

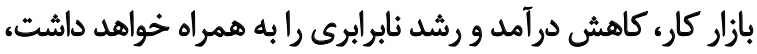

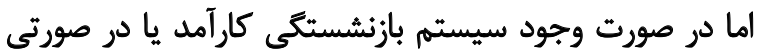

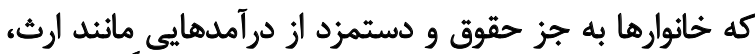

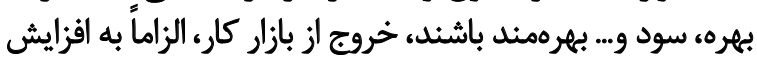

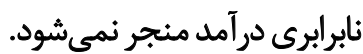

اكرجه مطالعات متعددى در زميئه عوامل مؤثر بر نابرابرى درآمد

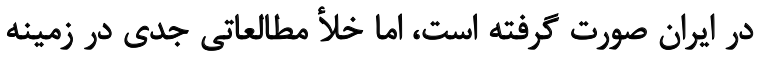

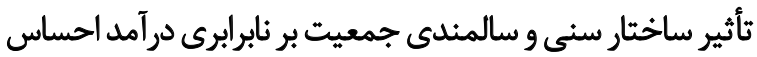

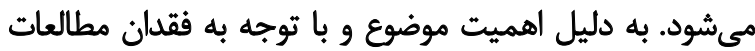

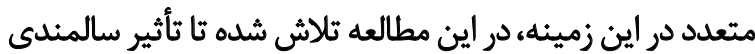

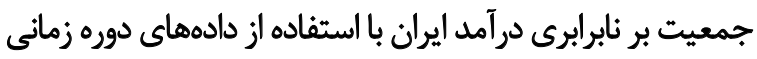

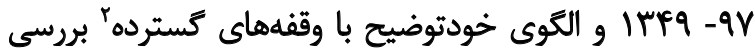

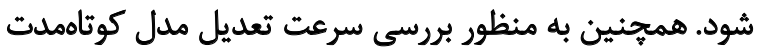

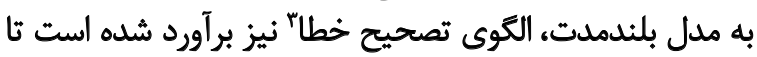

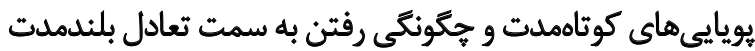

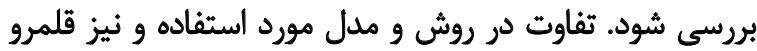
مكانى اين تحقيق را مى ثوان به عنوان نوآَورى آن قلمداد كرد

2. Auto Regressive Distributed Lag (ARDL)

3. Error Correction Model (ECM)
كردند. نتايج نشان داد كه سالمندى موجب كاهش سهمه منابع

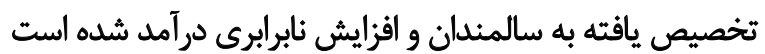

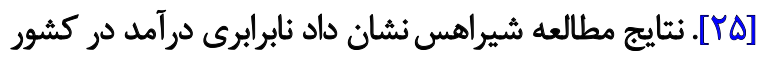

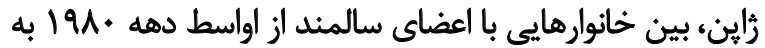

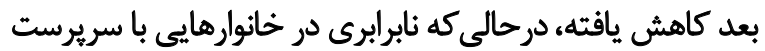

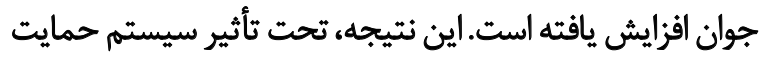

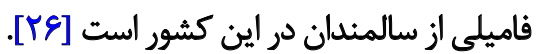
همجنين نتايج مطالعات انجامشده توسط بارت و همكاران،

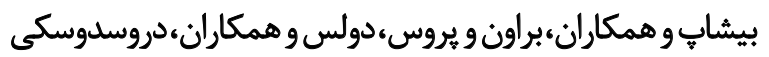

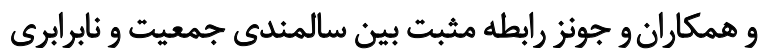

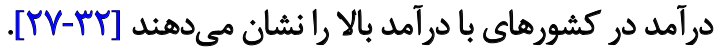

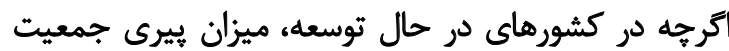

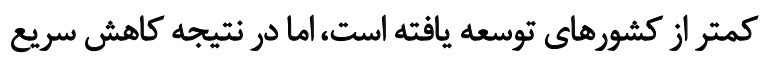

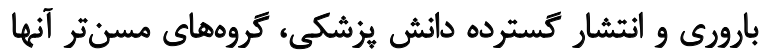

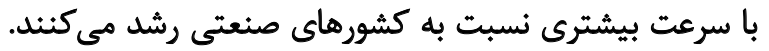

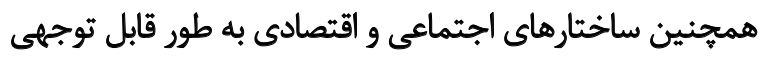

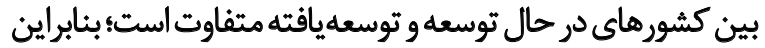

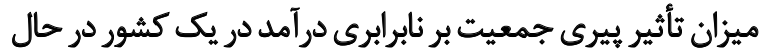

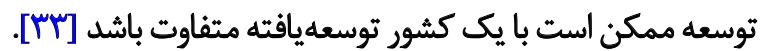

نتايج ئوهش انجامشده توسط زونى نشان داد يكى از على

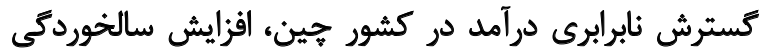

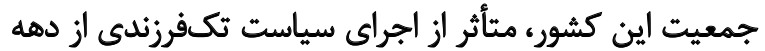

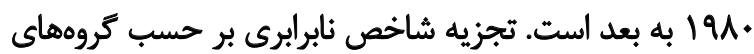

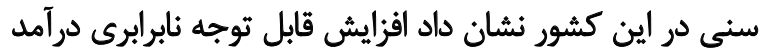

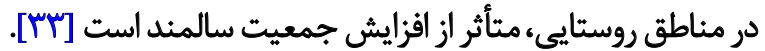

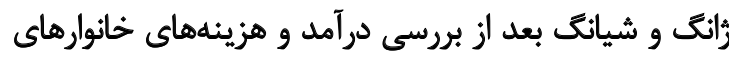

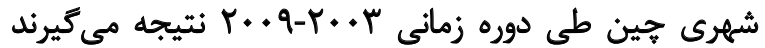

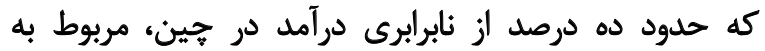

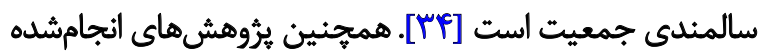

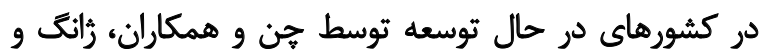

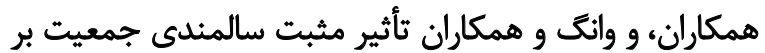

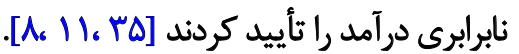
با اين حال، يُروهشهاى اندكى مانيد يُؤوهشهاى انجامشده

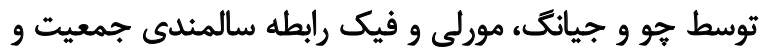

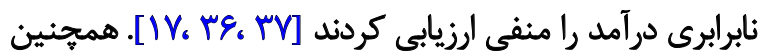

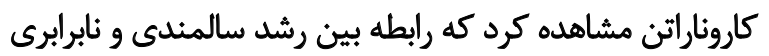

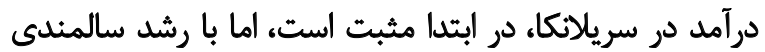

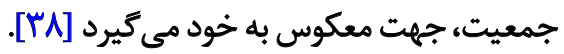

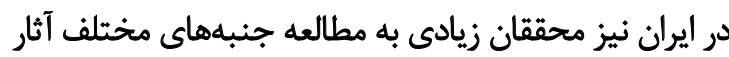

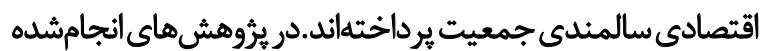

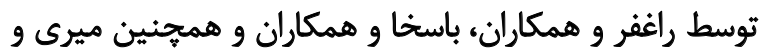

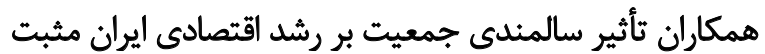


CPI (شاخص بهاى كالاها وخدمات مصرفى): رشد بهاى كالاها

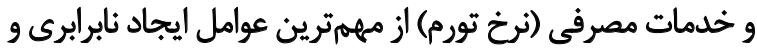

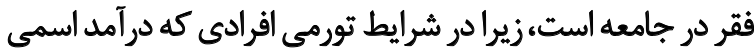

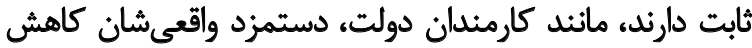

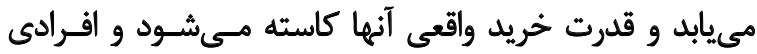

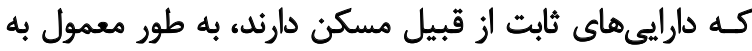

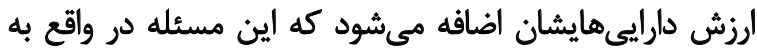

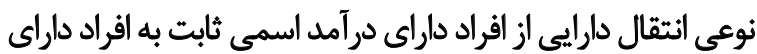

$$
\text { سـرمايه فيزيكى به شمار مىرودا }
$$

تpenness

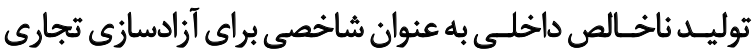

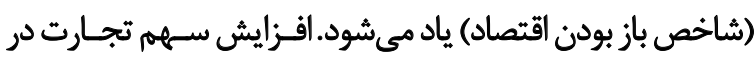

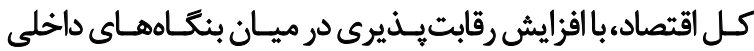

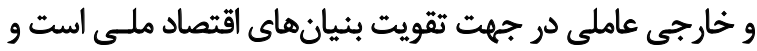
موجب ايجاد اشتغال و كاهش نابرابرى درآمد است.

شاخص GINI

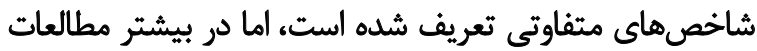

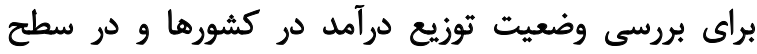

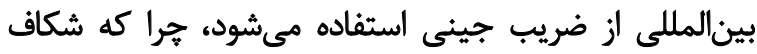

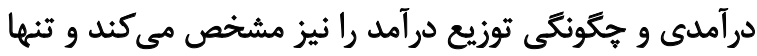

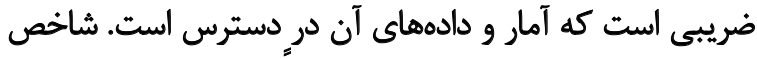

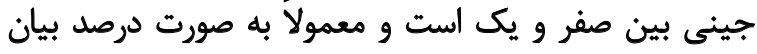

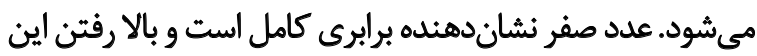
عدد به معناى نابرابرى بيشتر است.

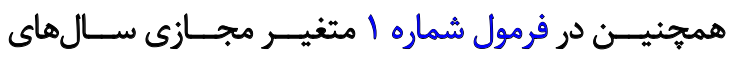

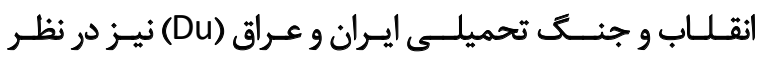

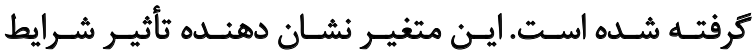

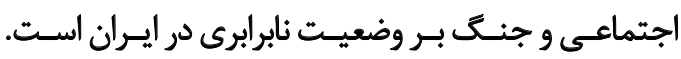

با توجه به اينكه فرمول شماره ا غيرخطي است، براي

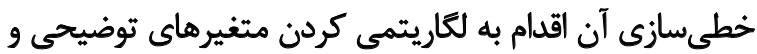

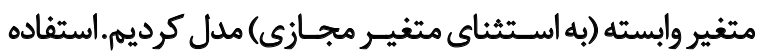

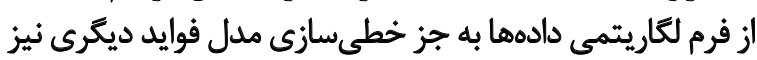

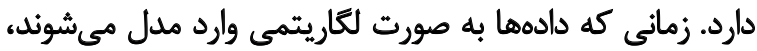

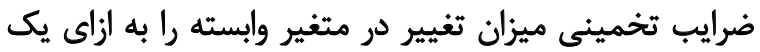

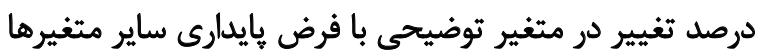

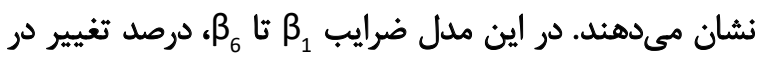

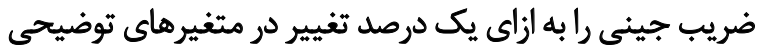

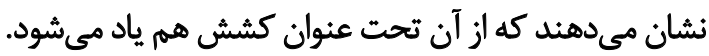

همجنين ضرايب بلدستآمده مستقل از واحد اندازهيرى نهي

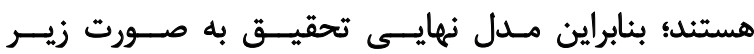

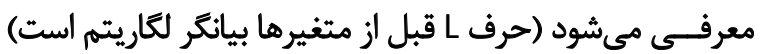

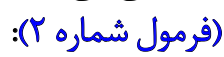

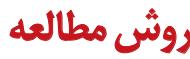

با توجه به اهميت موضوع سالمندى و تأثير آن بر نابرابرى

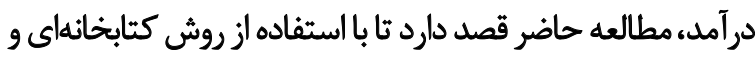

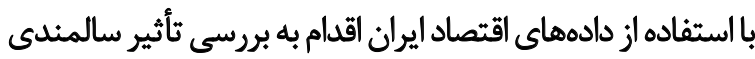

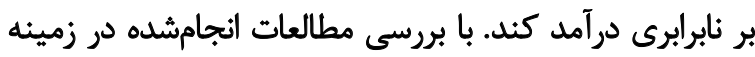

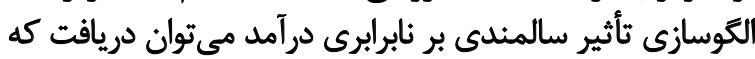

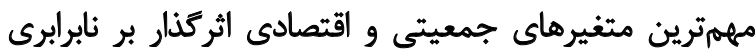

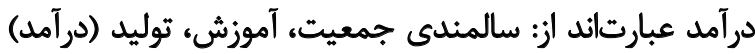

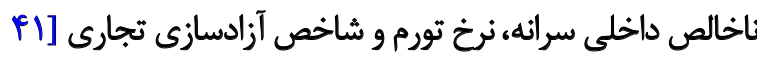

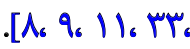

اكرجه معيارهاى مختلفى براى اندازمكيرى نابرابرى درآمد

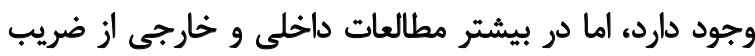

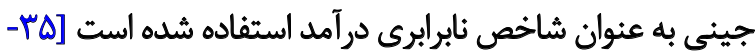

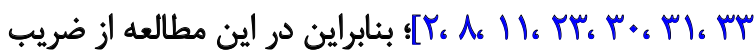

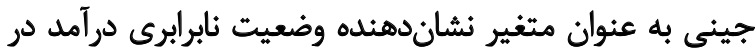

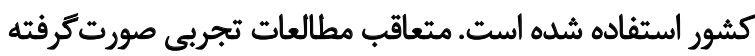

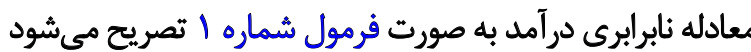

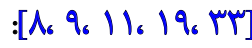

$G I N I_{t}=\alpha+b_{1}$ Ageing $_{t}+b_{2}$ Literacy $_{t}+b_{3} G D P_{t}+B_{4}\left(G D P_{t}\right.$ $1^{\wedge} 2+b_{5} \mathrm{CPI}_{t}+b_{6}$ Openness $_{t}+b_{7} D u+\varepsilon_{t}$

$$
\text { طورى كه در آن: }
$$

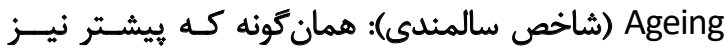

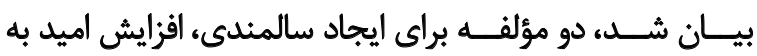

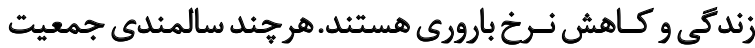

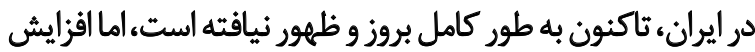

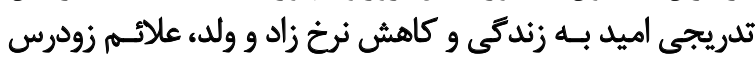

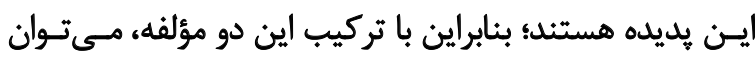

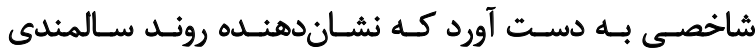

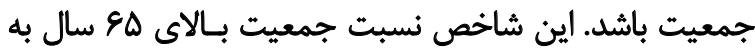

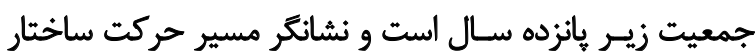
جمعيت در دهاهواي آتى است.

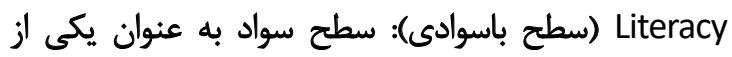

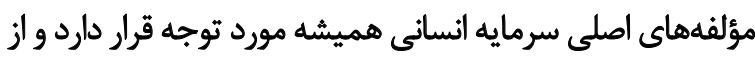

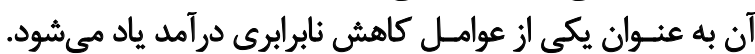

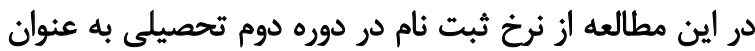
شاخصى براى سطح باسوادى استفاده شده است.

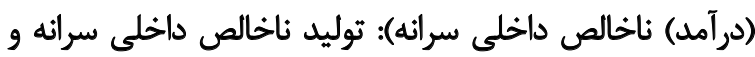

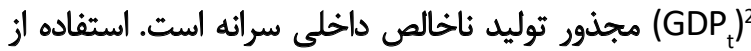

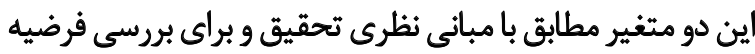

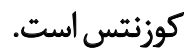




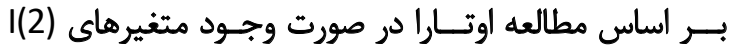

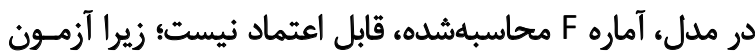

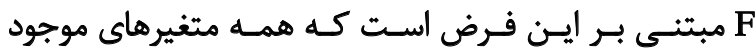

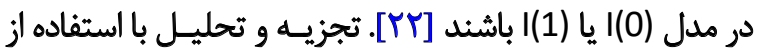

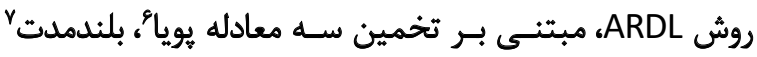

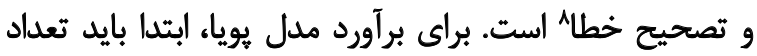

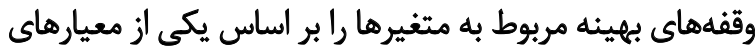

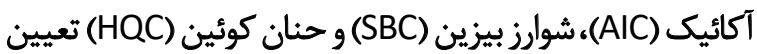

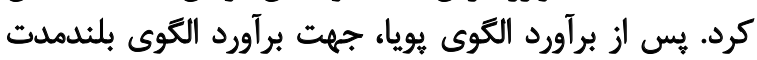

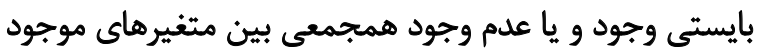

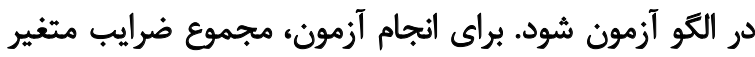

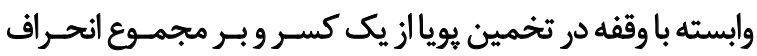

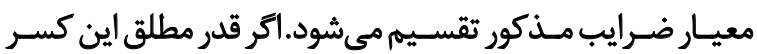

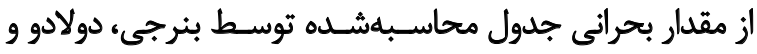

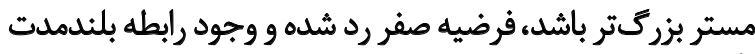

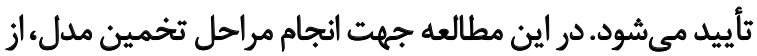
ترمافزار Eviews 10 استفاده مى شيود.

يافتهاها

آمار توصيفى متغيرها در جدول شماره ا ارائه شده است.

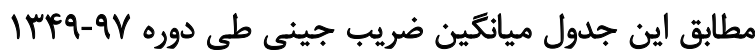

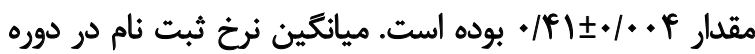

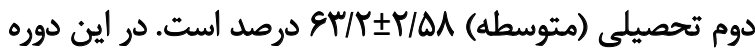

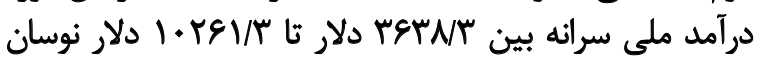

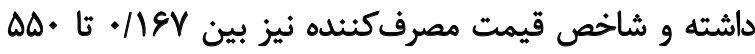

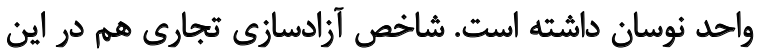

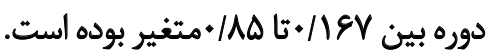

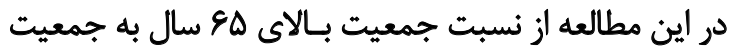

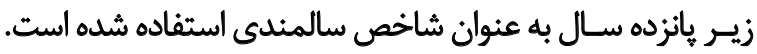

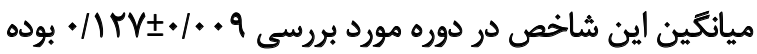

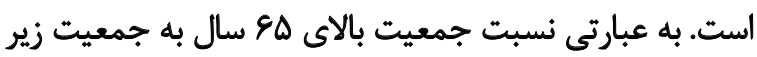

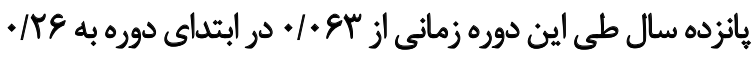

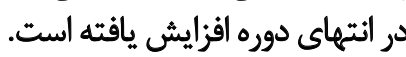

ابتدا به منظور جلو آنيرى از ركرسيون كاذب، مانايى دادهها

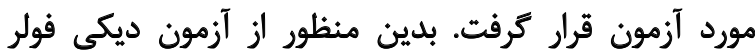

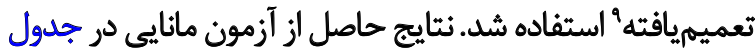
شماره r تزارش شده است. نتايج جدول شماره ب نشان مىدهد كه متغيرهاى لكاريتم

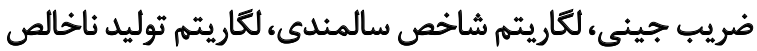

6. Dynamic

7. Long-run

8. Error-Correction

9. Augmated Dickey Fuller (ADF) .$r$

$\quad L G I N I_{t}=\alpha+B_{1} \quad$ LAgeing $_{t}+B_{2} \quad$ LLiteracy $_{t}+B_{3} \quad L G D P_{t}+B_{4}$ $\left(L G D P_{t}\right)^{2}+B_{5} L C P I_{t}+B_{6}$ LOpenness $_{t}+B_{7}$ Du $+\varepsilon_{t}$

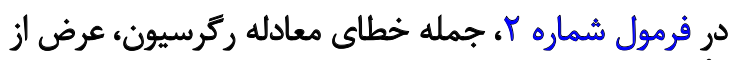

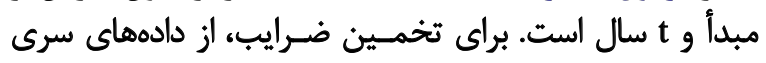

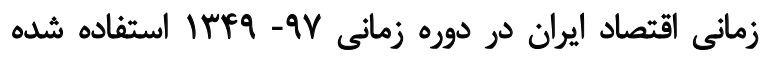

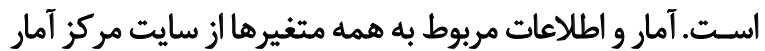

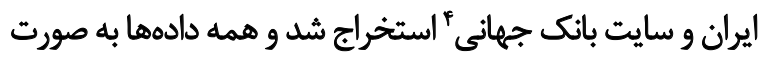
سالاثه هستئل.

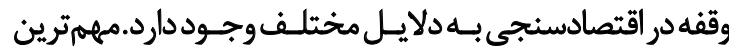

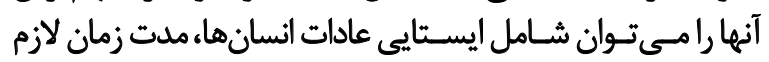

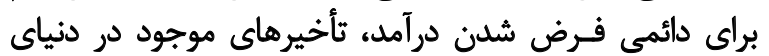

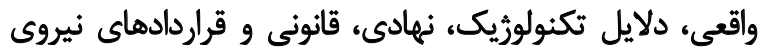

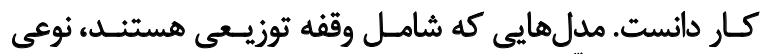

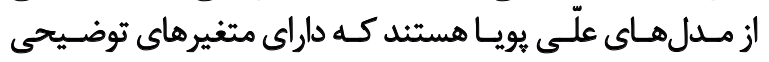

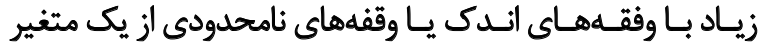
به عنوان متغيرهـاى توضيحى مورد استفاده قرار مى ئيرد. در اين وضسعيث احتمال وجود همخطسى ميـان متغيرهـا

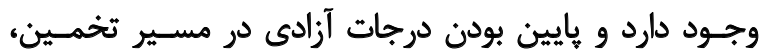

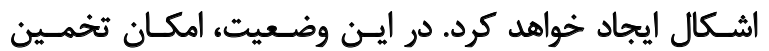

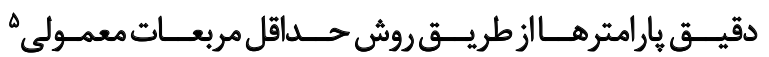

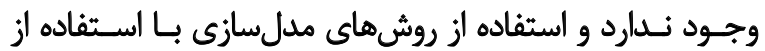

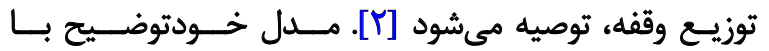

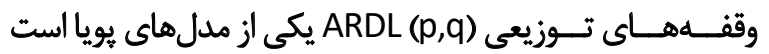

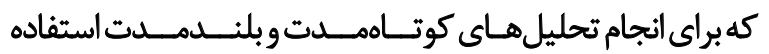

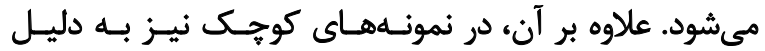

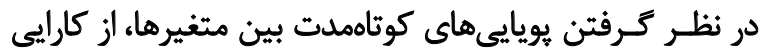

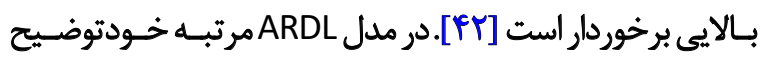

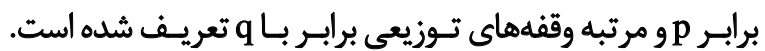

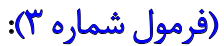

\section{.$r$}

$$
y_{t}=\alpha+b_{1} z_{1}+\sum_{i=1}^{p} B_{2 i} y_{t-j}+\sum_{j=0}^{q} B_{3 j} z_{t-j}+\varepsilon_{t}
$$

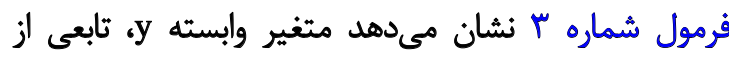

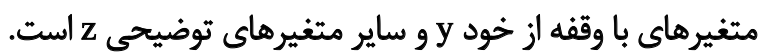

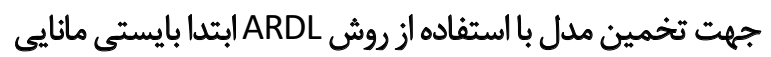

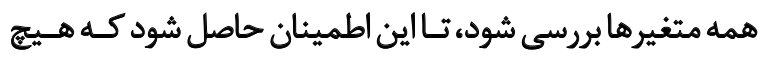

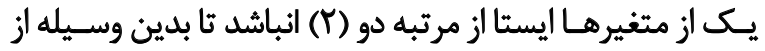
نتـايج سـاختكى اجتـــاب شـود.

4.World Development Indicators (WDI)

5. Ordinary Least Square 
جدول ا. آمار توصيفى متغيرها در ايران طى دوره زمانى IFF_-IFqV

\begin{tabular}{|c|c|c|c|}
\hline حداكثر & حداقل & ميانكَين||نحراف معيار & متغير \\
\hline 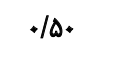 & $\cdot / T V$ & $.|f| \pm * / *+\varphi$ & GINI (شاخص نابرابرى درأمد) \\
\hline.$/ R$ & .1 .94 & $. / 1+r \pm+/ . .9$ & Ageing (شاخص سالمندى) \\
\hline N\&/A & re & $g T / Y \pm Y / \Delta A$ & Literacy \\
\hline $1 . r E V / T$ & PETN" & $\Delta q Y q / \Delta \pm T M T / \Delta$ & GDP \\
\hline$\Delta \Delta$ &.$/ 18 V$ & grtiv/N & (شاخص بهاى كالاها وخدمات مصرفى) CPI \\
\hline$\cdot / 1 \Delta$ &.$/ 4 \pi$ & $.10 \cdot \pm * / \cdot 19$ & Openness (شاخص أزادسازى تجارى) \\
\hline
\end{tabular}

N9 درصد از تغييرات متغير وابسته توسط متغيرهاي توضيحي،

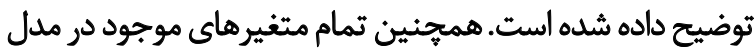

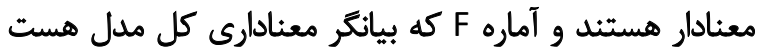

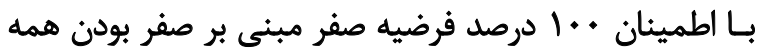

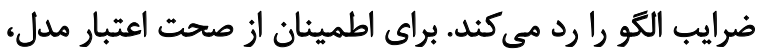

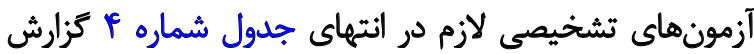

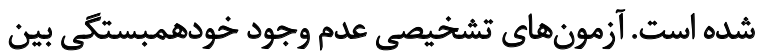

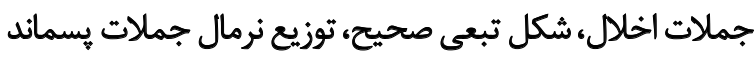

$$
\text { و همسانى واريانس را تأييد مى كنيد. }
$$

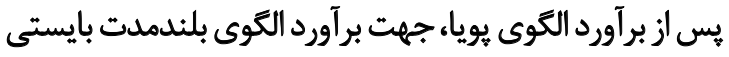

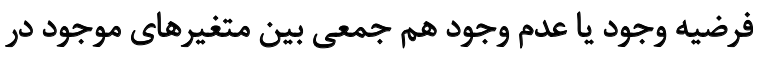

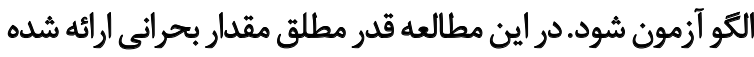

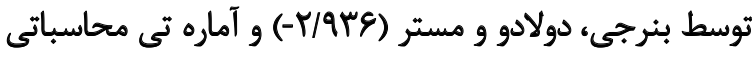

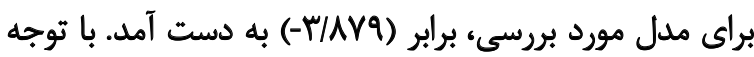

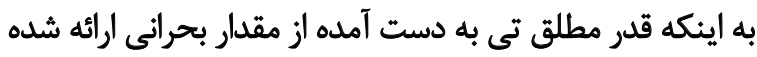

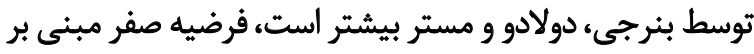

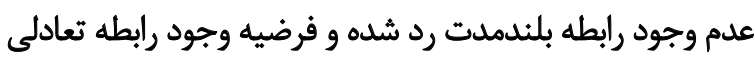

داخلم سرانه و لكاريتهم شاخص بهاي كالاها و خدمات مصرفي در

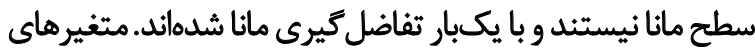

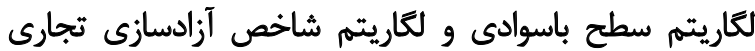

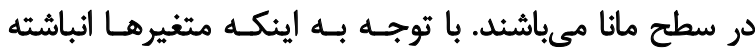

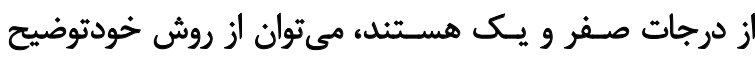
با وقفههاى كسترده (ARDL) براى تخمين مدل استفاده كرد.

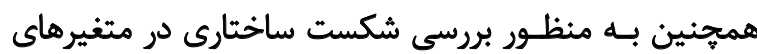

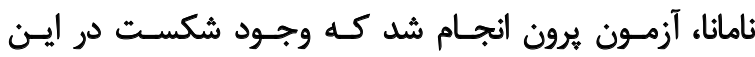

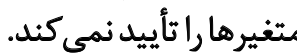

در اين مطالعه براى تعيين وقفه بهينه از معيار شوارز بيزين (SBC)

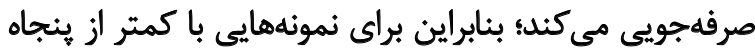

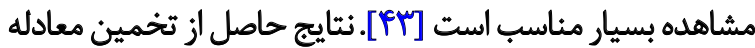

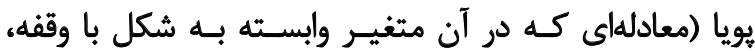

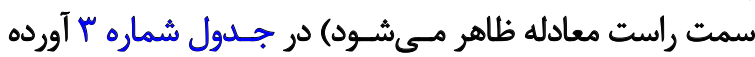

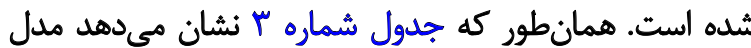
برآورد شده از R بالايى برخوردار است و به اين معناست كه

جدول r. نتايج آزمون مانيى بر اساس آماره ديكى قولر تعميميافته

\begin{tabular}{|c|c|c|c|c|c|c|}
\hline \multicolumn{3}{|c|}{ باعرض از مبدأ وروند } & \multicolumn{3}{|c|}{ باعرض از مبدأ و بدون روند } & \multirow[b]{2}{*}{ متغير } \\
\hline وضعيت مانايي & سطدار بحرانى در مر مد & آماره آزمون & وضعيت مائايى & 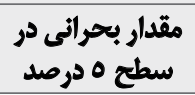 & آماره آزمون & \\
\hline$I(1)$ & $-r / r \mid$ & $-r / M^{e}$ & (I) 1 & $-r / \cdot 1$ & $-r / q$. & $\operatorname{LGINI}_{\mathrm{t}}$ \\
\hline $\mid(1)$ & $-r / 10$ & $-8 / 8 \Delta$ & (I) & $-r / q u$ & $-r / R t$ & LAgeing $_{t}$ \\
\hline$I(0)$ & $-r / \Delta)$ & $-F / T V$ & (I) & $-r / q r$ & $-F / \Delta V$ & LLiteracy $_{\mathrm{t}}$ \\
\hline $\mid(1)$ & $-r / r)$ & $-r / \& 8$ & (I) 1 & $-r / q r$ & $-r / 48$ & $\mathrm{LGDP}_{\mathrm{t}}$ \\
\hline$I(1)$ & $-4 / .9$ & $-p / p$ & (I) 1 & $-r / 9 Y^{w}$ & $-r / v$ & $\mathrm{LCPI}_{\mathrm{t}}$ \\
\hline$I(0)$ & $-r / \Delta$. & $-\Delta / m$ & (I). & $-r / 9 r$ & $-\Delta / T T$ & LOpenness $_{t}$ \\
\hline
\end{tabular}


جدول ب. نتابج برآورد رابطه يويا (1,1,0,1,0,1,1,0

\begin{tabular}{|c|c|c|}
\hline أماره تى [اجتمال]] & ضريب & متغير \\
\hline$T / M E[\% / \% . r]$ &.$|f \Delta|$ & LGINI(-1) \\
\hline $1 / 1 \Delta H[\cdot 1.8 *]$ & 吾 & LAgeing $_{\mathrm{t}}$ \\
\hline$Y / * F \Delta[\%+\% \Lambda]$ & .1 .18 & LAgeing_t (-1) \\
\hline$-r / r I F[\cdots \cdots]$ &.$- / T M$ & LLiteracy $_{t}$ \\
\hline$r / 9)[\% / \%$ H $]$ &.$/ r i r$ & $\operatorname{LGDP}_{t}$ \\
\hline t/RTt [*/.r. $]$ & ./NG & $\operatorname{LGDP}_{t}(-1)$ \\
\hline$-1 / N+2[\cdot / \cdot \Delta \cdot]$ &.$- / 1 r V$ & $\left(\text { LGDP }_{t}\right)^{2}$ \\
\hline 1/qux $[\% / \cdot \pi T]$ & $\%$ & LCPI_t \\
\hline$V / M \cdot[\% / . \& 1]$ & $. \cdots+4$ & $\operatorname{LCPI}_{t}(-1)$ \\
\hline 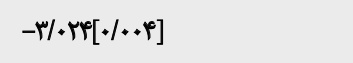 & $-.1 . .1$ & LOpenness $_{t}$ \\
\hline$-Y / R W[\cdot / \cdot \cdot 1]$ &.$- / \cdot .14$ & LOpenness $_{t}(-1)$ \\
\hline $1 / F+Y[\cdot / \pi T 1]$ & $\%+r$ & Du \\
\hline$-\cdot / q \cdot \Delta[\cdot / r V)]$ & - T/Rat & c \\
\hline R-Squared $=\cdot 1 \wedge q$ & & Adjusted R-Squared $=\cdot / \wedge \Delta$ \\
\hline$D . W=r / * r Y$ & & $\mathrm{~F}=\mathrm{TYE} / \mathrm{N}[\bullet+* / \bullet]$ \\
\hline
\end{tabular}

جدول F. نتايج برآورد ضرايب تصحيح خطا (1,1,0,1,0,1,1,0

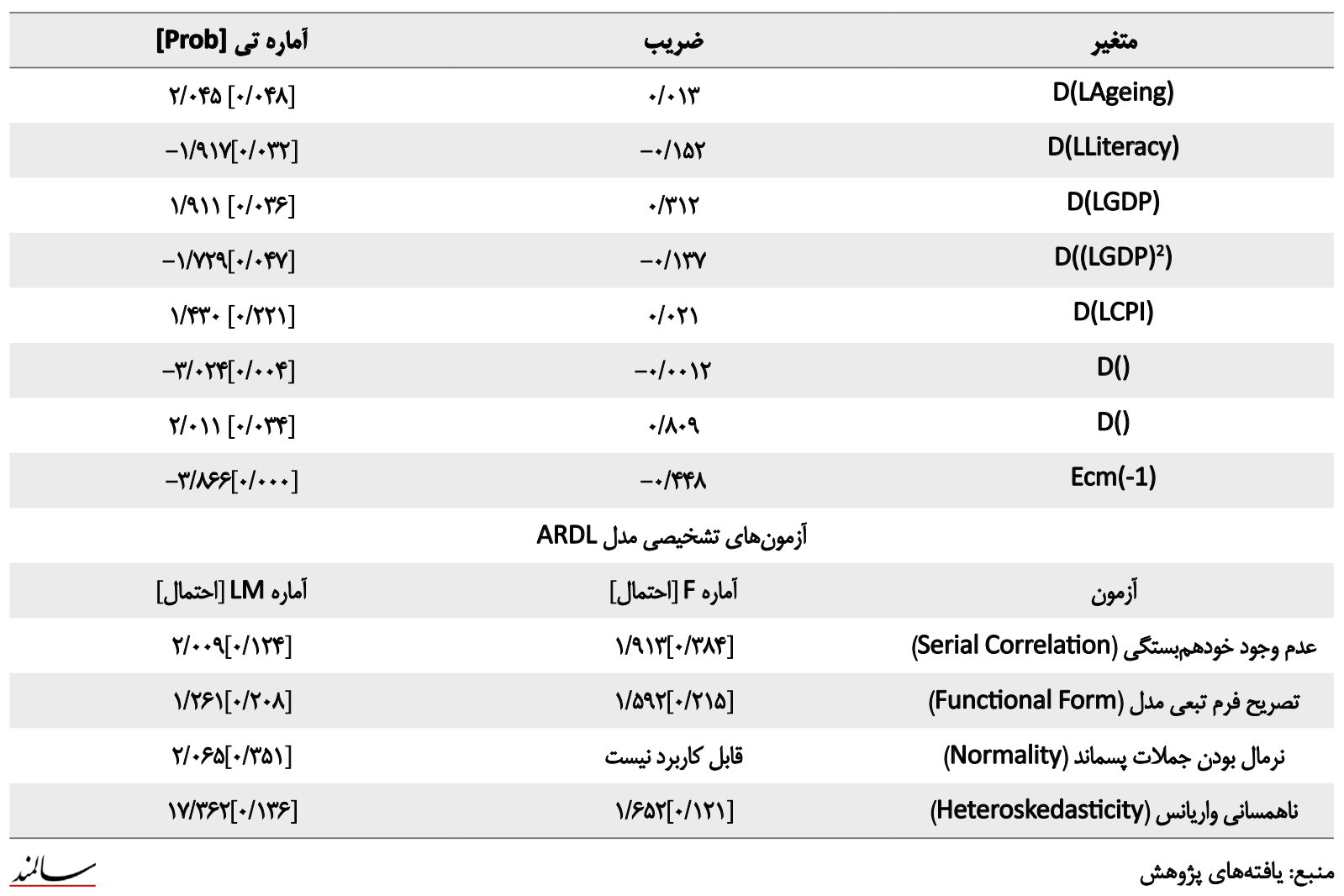


جدول ه. نتايج برآورد رابطه بلندمدت (1,1,0,1,0,1,1,0

\begin{tabular}{|c|c|c|}
\hline آماره تى [احتمال]] & ضرايب & متغير \\
\hline $1 / \wedge q 4[\cdot / \cdot+4]$ &.$/ N F$ & LAgeing \\
\hline$-r / F \cdot 1[\cdot / \cdot r]$ &.$- / 197$ & LLiteracy \\
\hline$T / M E[.1 . .1]$ & . Narr & LGDP \\
\hline$-1 / \Lambda \cdot 9[\cdot / \cdot+\mu r]$ &.$- /$ TOII & $(\text { LGDP) })^{2}$ \\
\hline$-1 / 91+[. / .49]$ &.$- /+$ MIf & $\mathrm{LCPI}$ \\
\hline$-1 / A F q[\cdot / \cdot \mid \Lambda]$ & $-.1+.84$ & LOpenness \\
\hline$r / \Delta \Delta[\% / \pi]$ & $. / 1 \mid A$ & $\mathrm{Du}$ \\
\hline$-\cdot /$ A $F[\cdot / f \cdot 1]$ & - - lafar & C \\
\hline
\end{tabular}

جن و همكاران و همجنين دالس و همكاران كه نشان مى دهند

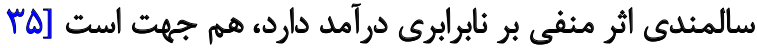

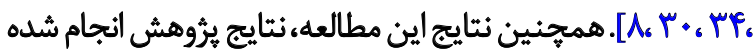

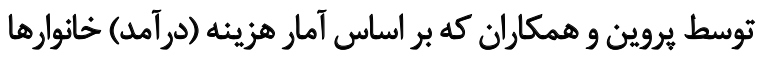

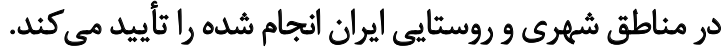
آموزش و باسوادى هم به عنوان متغير اصلى تشكيل دهنده

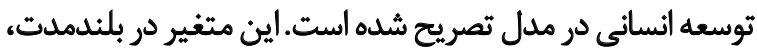

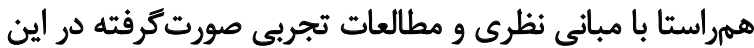

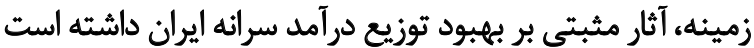

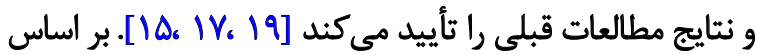

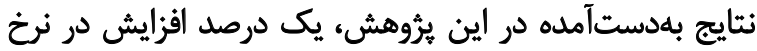

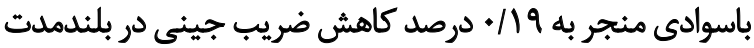

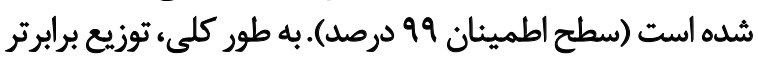

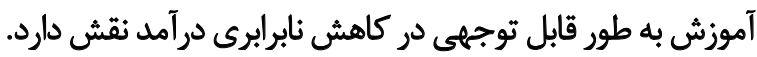

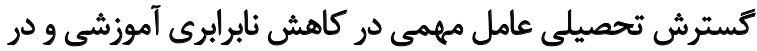
نتيجه نابرابرى درآمد است.

توليد ناخالص داخلي سرانه در بلندمدت تأثير منفى بر توزيع

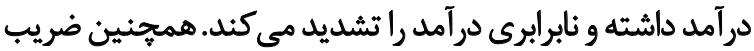

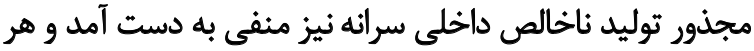

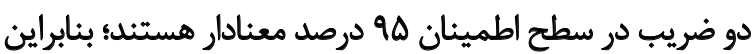

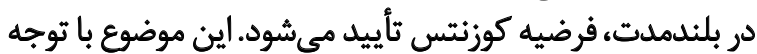

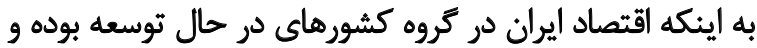

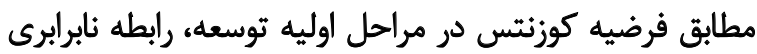

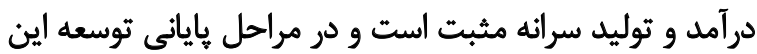

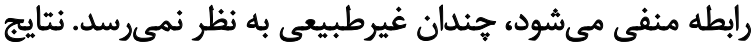

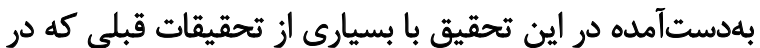

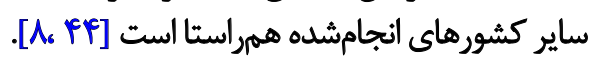

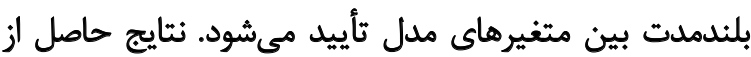
تخمين رابطه بلندمدت در جدول شماره ها آورده شده است.

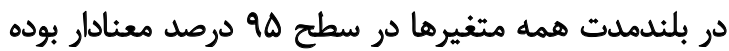

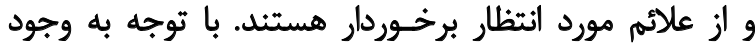

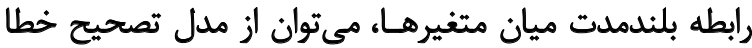

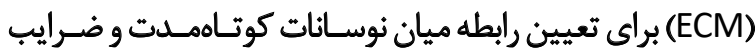

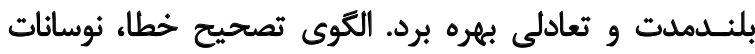

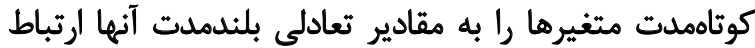

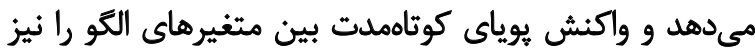

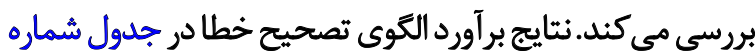
أورده شده است.

$$
\text { بְ }
$$

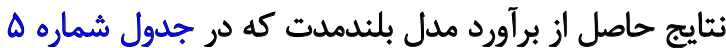

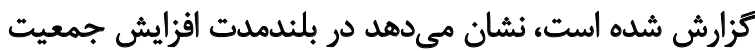

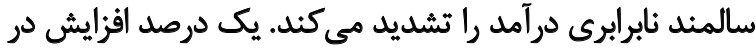

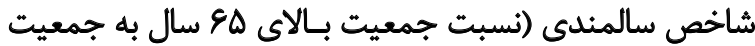

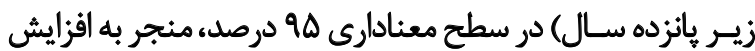

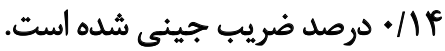

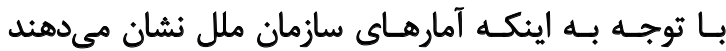

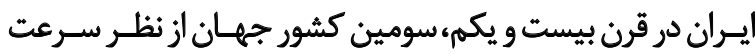

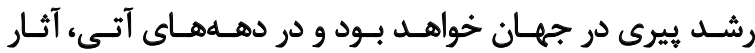

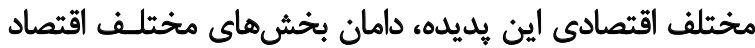

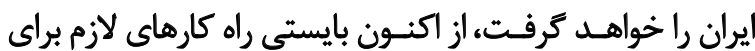

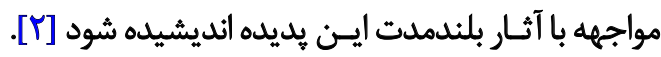

نتايج بهدستآمده در اين تحقيق با بسيارى از تحقيقات

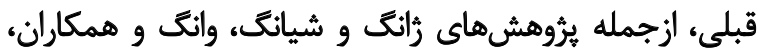




\section{نتيجه كيرى نهايى}

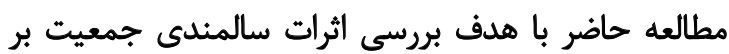

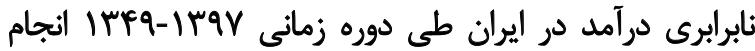

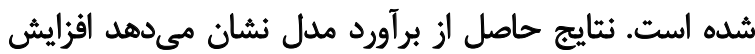

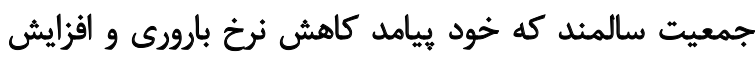

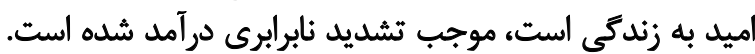

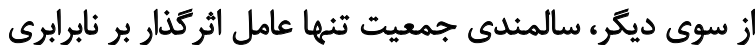

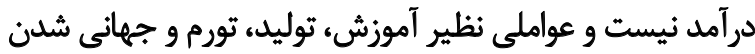

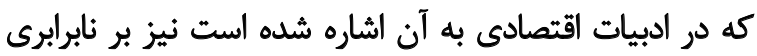

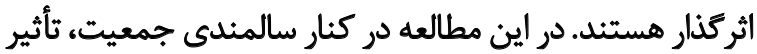

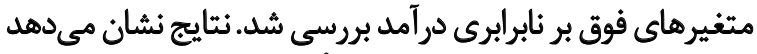

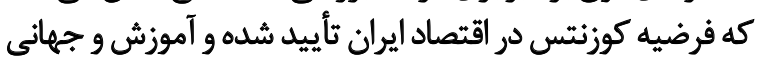

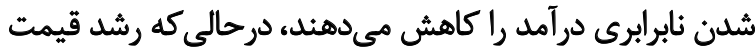

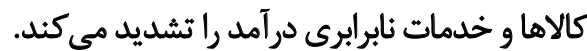

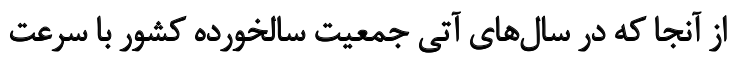

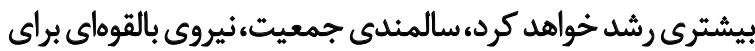

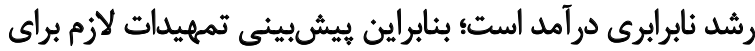

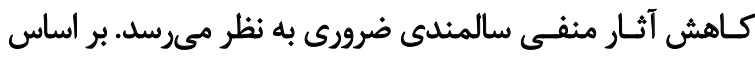

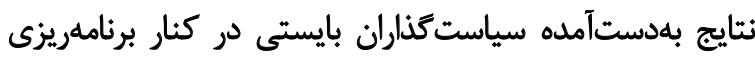

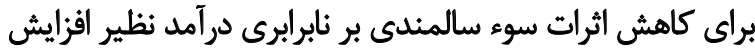

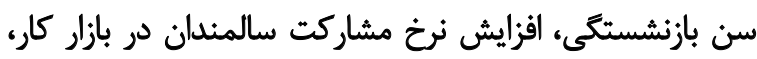

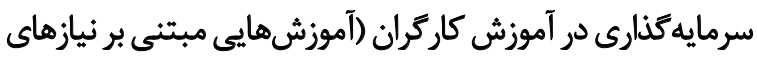

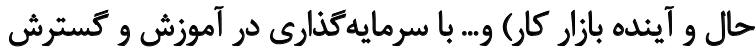

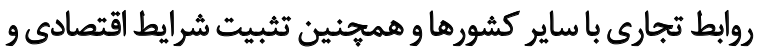

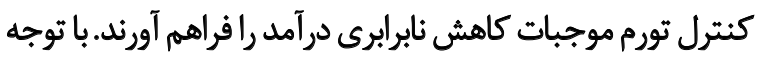

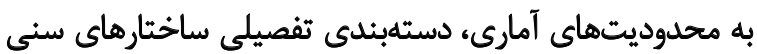

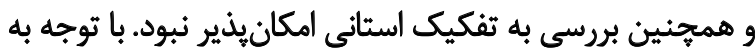

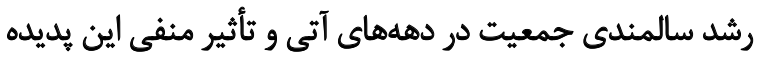

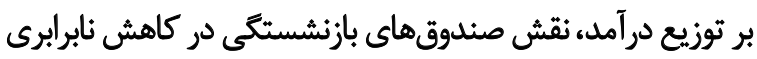

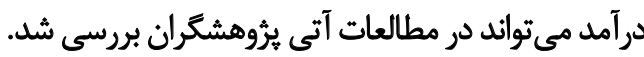

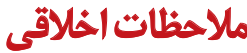

\section{ي ميروى أز اصول اخلاق يثوهش}

اين مقاله مورد تاييد كميته اخلاق دانشكاه تربيت مدرس قرار كرفته است (كد: 1R.MP.127).

$$
\text { كامي مالى }
$$

اين بُروهش بخشى از رساله دكترى نويسنده اول در كروه

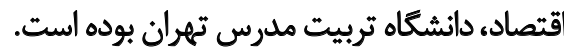

ضريب متغير شاخص بهاي كالاها و خدمات مصرفي نيز در

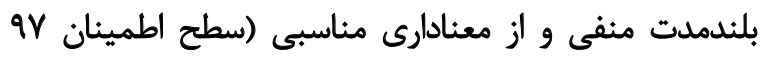

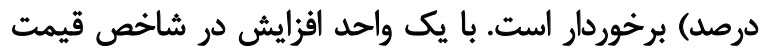

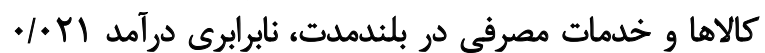

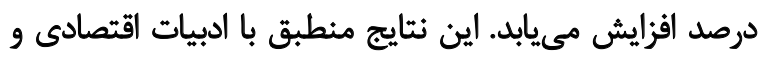

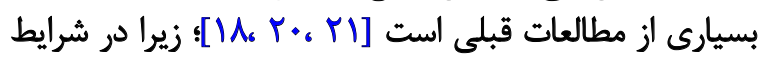

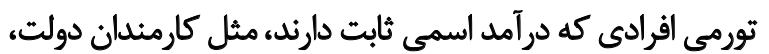

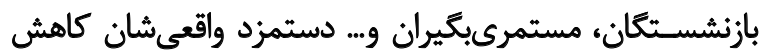

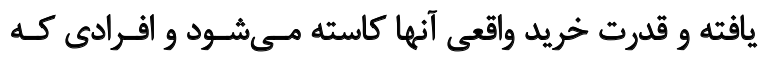

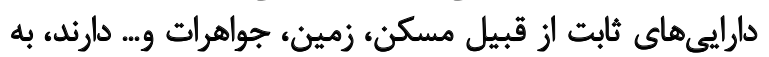

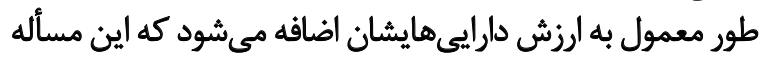

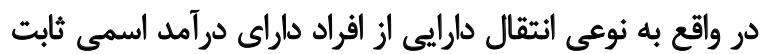

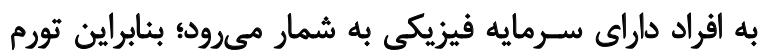
مى تواند به افزايش شكاف درآ فيدى منجر شود.

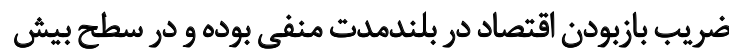

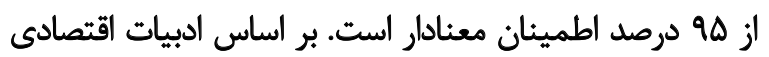

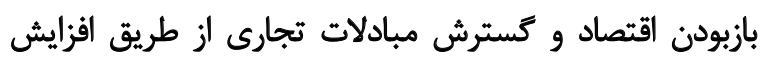

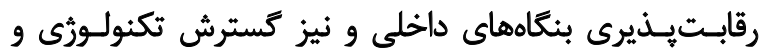

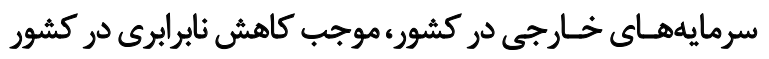

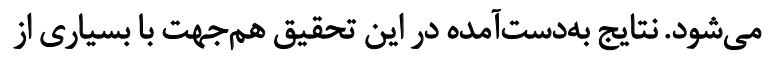

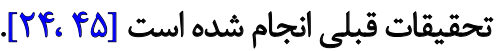

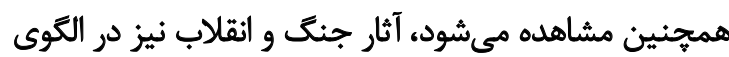

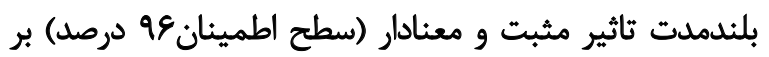

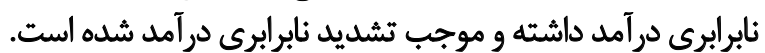

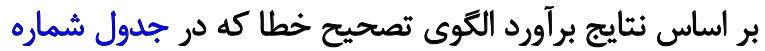

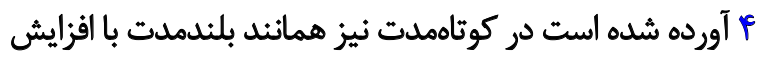

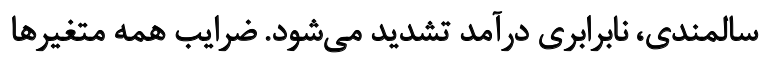

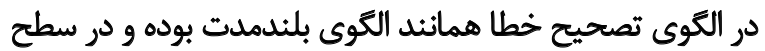
الطمينان ه9 درصد معنادار هستيند.

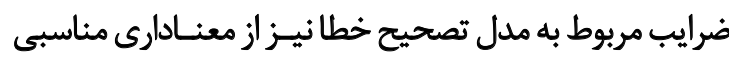

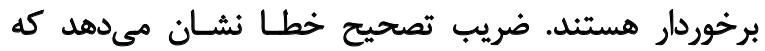

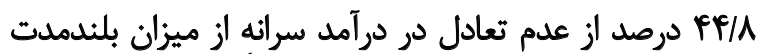

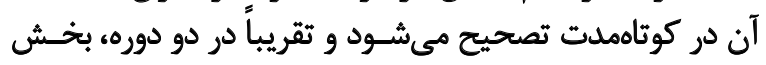

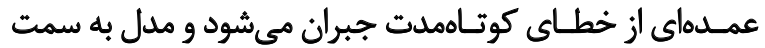

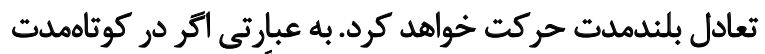

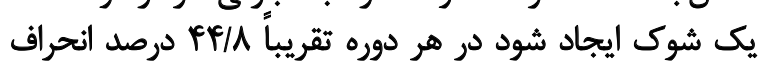

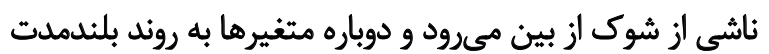

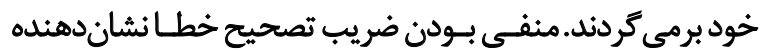

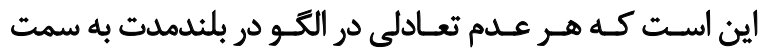

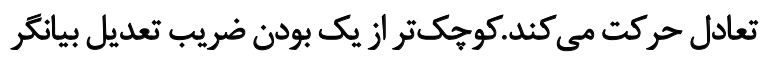
همكرايى مدل در كوتامدت است 


$$
\text { مشار كت نويسندكّان }
$$

$$
\begin{aligned}
& \text { تمام نويسندكان در طراحي، اجرا و نكارش همه بخشهاى } \\
& \text { يُوروه حاضر مشاركت داشتهاند. } \\
& \text { ثعارض منافع }
\end{aligned}
$$

در اين مقاله هيجگونه تعارض منافعى وجود ندارد. 


\section{References}

[1] Yavari K, Basakha M, Sadeghi H, Naseri AR. [Economic aspects of ageing (Persian)]. Salmand: Iranian Journal of Ageing. 2015; 10(1):92-105. http:/ / salmandj.uswr.ac.ir/article-1-702-en.html

[2] Basakha M, Yavari K, Sadeghi H, Naseri A. [Population aging and Iran's non-oil economic growth (Persian)]. Payavard Salamat. 2015; 9(2):131-46._http:// payavard.tums.ac.ir/article-1-5689-en. $\mathrm{html}$

[3] Mirzaie M, Darabi S, Babapour M. [Population aging in Iran and rising health care costs (Persian)]. Salmand: Iranian Journal of Ageing. 2017; 12(2):156-69. [DOI:10.21859/sija-1202156]

[4] Miri N, Maddah M, Raghfar H. [Aging and economic growth (Persian)]. Salmand: Iranian Journal of Ageing. 2019; 13(5):626-37. [DOI:10.32598/SIJA.13.Special-Issue.626]

[5] Parvin S, Bagheri Pormehr Sh, Pagard Alishahi F. [The affect of population aging on income inequality in Iran (Persian)]. Population Studies. 2019; 5(1):103-25. http://jips.psri.ac.ir/article_108262.html?lang=en

[6] Alvaredo F. World inequality report 2018. Cambridge, MA/London: Harvard University Press; 2018. [DOI:10.4159/9780674984769]

[7] Nili M, Frahbakhsh A. [The relationship between economic growth and income distribution (Persian)]. The Journal of Planning and Budgeting. 1999; 3(10-11):121-54. http://jpbud.ir/article-1-244-fa.html

[8] Chen X, Huang B, Li S. Population aging and inequality: Evidence from China. The World Economy. 2018; 41(8):1976-2000. [DOI:10.1111/twec.12551]

[9] Gustafsson B, Johansson M. In search of smoking guns: What makes income inequality vary over time in different countries? American Sociological Review. 1999; 64(4):585-605. [DOI:10.2307/2657258]

[10] Kuznets S. Economic growth and income inequality. The American Economic Review. 1955; 45(1):1-28. https:/ / www.jstor. org/stable/1811581

[11] Zhang H, Ke L, Ding D. The effect of chinese population aging on income inequality: Based on a micro-macro multiregional dynamic cge modelling analysis. Emerging Markets Finance and Trade. 2021; 57(5):1399-419. [DOI:10.1080/154049 6X.2019.1623781]

[12] Piketty T. Capital and wealth taxation in the 21st century. National Tax Journal. 2015; 68(2):449-58. [DOI:10.17310/ntj.2015.2.10]

[13] Malthus TR. First essay on population, 1798. London: Palgrave Macmillan; 1966. https:/ /link.springer.com/book/10.1007/9781-349-81729-0

[14] Dallali Esfahani R, Esmaeilzadeh R. [A new view on population ideas (Malthus, Keynes and Becker Revisions) (Persian)]. Journal of Social Sciences. 2007; 4(1):97-120. [DOI:10.22067/JSS.V0I0.8719]

[15] Barro RJ. Inequality and growth in a panel of countries. Journal of Economic Growth. 2000; 5(1):5-32. [DOI:10.1023/A:1009850119329]

[16] Faik J. Impacts of an ageing society on macroeconomics and income inequality - the case of Germany since the 1980s. SOEPpaper. 2012; Desember;No. 518. [DOI:10.2139/ssrn.2190302]
[17] De Gregorio JD, Lee JW. Education and income inequality: New evidence from cross_country data. Review of Income and Wealth. 2002; 48(3):395-416. [DOI:10.1111/1475-4991.00060]

[18] Law CH, Soon SV. The impact of inflation on income inequality: the role of institutional quality. Applied Economics Letters. 2020; 27(21):1735-8. [DOI:10.1080/13504851.2020.1717425]

[19] Lee JW, Lee H. Human capital and income inequality. Journal of the Asia Pacific Economy. 2018; 23(4):554-83. [DOI:10.1080/13 547860.2018.1515002]

[20] Monnin P. Inflation and income inequality in developed economies. CEP Working Paper Series. 2014; June. [DOI:10.2139/ ssrn.2444710]

[21] Muhibbullah M, Das MR. The impact of inflation on the income inequality of Bangladesh: A time series analysis. International Journal of Business and Technopreneurship. 2019; 9(2):141-50. http://103.86.130.60/handle/123456789/61023

[22] Ouattara B. Foreign aid and fiscal policy in Senegal. Manchester, UK: University of Manchester; 2004.

[23] van der Ploeg F. Natural resources: Curse or blessing? Journal of Economic literature. 2011; 49(2):366-420. [DOI:10.1257/jel.49.2.366]

[24] Wu JY, Hsu CC. Foreign direct investment and income inequality: Does the relationship vary with absorptive capacity? Economic Modelling. 2012; 29(6):2183-9. [DOI:10.1016/j.econmod.2012.06.013]

[25] Gruber J, Wise D. An international perspective on policies for an aging society. NBER Working Paper. 2001; January:No. 8103. [DOI:10.3386/w8103]

[26] Shirahase S. Income inequality among older people in rapidly aging Japan. Research in Social Stratification and Mobility. 2015 41:1-10. [DOI:10.1016/j.rssm.2015.03.001]

[27] Barrett GF, Crossley TF, Worswick C. Consumption and income inequality in Australia. Economic Record. 2000; 76(233):116-38. [DOI:10.1111/j.1475-4932.2000.tb00011.x]

[28] Bishop JA, Formby JP, Smith WJ. Demographic change and income inequality in the United States, 1976-1989. Southern Economic Journal. 1997; 64(1):34-44. [DOI:10.1002/j.2325-8012.1997. tb00003.x] [PMID]

[29] Brown RL, Prus SG. Income inequality over the later-life course: A comparative analysis of seven OECD countries. Annals of Actuarial Science. 2006; 1(2):307-17. [DOI:10.1017/S1748499500000178]

[30] Dolls M, Doorley K, Paulus A, Schneider H, Sommer E. Demographic change and the European income distribution. The Journal of Economic Inequality. 2019; 17(3):337-57. [DOI:10.1007/ s10888-019-09411-z]

[31] Drosdowski T, Stöver B, Wolter MI. The impact of ageing on income inequality. GWS Discussion Paper Series. 2015; 15-16. https://ideas.repec.org/p/gws/dpaper/15-16.html

[32] Jones RS. Income inequality, poverty and social spending in Japan. OECD Economics Department Working Papers. 2007; No. 556. https://www.oecd-ilibrary.org/economics/income-inequality-poverty-and-social-spending-in-japan_177754708811

[33] Zhong $\mathrm{H}$. The impact of population aging on income inequality in developing countries: Evidence from rural China. China Economic Review. 2011; 22(1):98-107. [DOI:10.1016/j.chieco.2010.09.003] 
[34] Zhang J, Xiang J. How aging and intergeneration disparity influence consumption inequality in China. China \& World Economy. 2014; 22(3):79-100. [DOI:10.1016/j.chieco.2010.09.003]

[35] Wang Ch, Wan G, Luo Zh, Zhang X. Aging and inequality: The perspective of labor income share. ADBI Working Paper. 2017; No. 764. https://www.econstor.eu/handle/10419/179220

[36] Chu CYC, Jiang L. Demographic transition, family structure, and income inequality. The Review of Economics and Statistics. 1997; 79(4):665-9. [DOI:10.1162/003465397557079]

[37] Morley SA. The effect of changes in the population on several measures of income distribution. The American Economic Review. 1981; 71(3):285-94. https://www.jstor.org/stable/1802779

[38] Karunaratne HD. Age as a factor determining income inequality in Sri Lanka. The Developing Economies. 2000; 38(2):211-42. [DOI:10.1111/j.1746-1049.2000.tb00877.x]

[39] Raghfar H, Mousavi MH, Ardalan Z. [Aging phenomenon impacts and efficiency changes effects on pension and macroeconomic variables using a dynamic general equilibrium approachover lapping generation model (OLG) (Persin)]. Journal of Population Association of Iran. 2014; 9(17):7-35. http:/ / www.jpaiassoc. ir/article_20067.html

[40] Dong Zh, Tang C, Wei X. Does population aging intensify income inequality? Evidence from China. Journal of the Asia Pacific Economy. 2018; 23(1):66-77. [DOI:10.1080/13547860.2017.135427]

[41] Lui HK. Ageing population and rising income inequality in post-handover Hong Kong. Review of Integrative Business and Economics Research. 2019; 8(1):51-63. http:/ / buscompress.com/ uploads/3/4/9/8/34980536/riber_8-1_x01_b18-028_51-63.pdf

[42] Ahmad M, Tashkini A, Soori AR. [The estimation of consumption function in Iran's economy (Persian)]. Economics Research. 2008; 8(28):15-39. https://joer.atu.ac.ir/article_3230.html

[43] Noferesti M. [Demographic changes and demand for money in Iran (Persian)]. Ravand. 2011; 19(58):15-32. https://www.cbi. ir/page/8353.aspx

[44] Saith A. Development and distribution: A critique of the crosscountry U-hypothesis. Journal of Development Economics. 1983; 13(3):367-82. [DOI:10.1016/0304-3878(83)90006-8]

[45] Asteriou D, Dimelis S, Moudatsou A. Globalization and income inequality: A panel data econometric approach for the EU27 countries. Economic Modelling. 2014; 36:592-9. [DOI:10.1016/j. econmod.2013.09.051] 
This Page Intentionally Left Blank 